International Journal of Engineering \& Technology, $7(1.1)(2018) 439-443$
SPC
International Journal of Engineering \& Technology
Website www.sciencepubco.com/index.php/IJET
Research paper

\title{
Neighbordiscovery-based security enhancement using threshold cryptography for IP address assigning in network
}

\author{
R. Bharanidharan ${ }^{1 *}$, R. Santhosh ${ }^{2}$ \\ ${ }^{1}$ Research Scholar, Department of CSE, Karpagam Academy of Higher Education, Coimbatore, Tamilnadu, India \\ ${ }^{2}$ Associate Professor, Department of CSE, Karpagam Academy of Higher Education, Coimbatore, Tamilnadu, India
}

\begin{abstract}
The security threats related to current IP configurationand also includes with Threshold Cryptography (TC). Circulated situations are attractive more prevalent as these knowledge such as networks, aim to enable a large scale collaboration for resource sharing outline in the network. Secure verification is the interesting concern for such surroundings. The proposed system to maintain a two stages for improving the network security based on IP addressing time in the network. An first stage is threshold cryptography and another one is Neighbor discovery protocol (NDP). In main objectives of our proposed work is Threshold Cryptography based addressing scheme in network, also the authorization process fully depends on Neighbor discovery protocol based frameworks. The goal of the Neighbor discovery protocol is to support a Mobile Node (MN) roaming across network fields without communication disturbance or recognizable delay. Moreover, this approach also ensures correct synchronization between nodes that send overlapping data on the network. Finally, using this NDP-TC during data transfer time packet loss is very low so the network security is automatically increased.
\end{abstract}

Keywords:Threshold Cryptography; Encryption and Decryption; NDP; Security.

\section{Introduction}

A wireless network is required for infrastructure mode wireless networking. Hence it offers the benefit of scalability, consolidated security controlling and better connectivity. In the ad hoc mode of wireless networks, the nodes can directly communicate with each other without using any access point [1-3]. To set up a specially appointed wireless system, every remote connector must be designed for unplanned mode versus the framework mode. An network system tends to include a little gathering of gadgets all in closeness to each other. In this system the execution corrupts as the quantity of hubs increments.

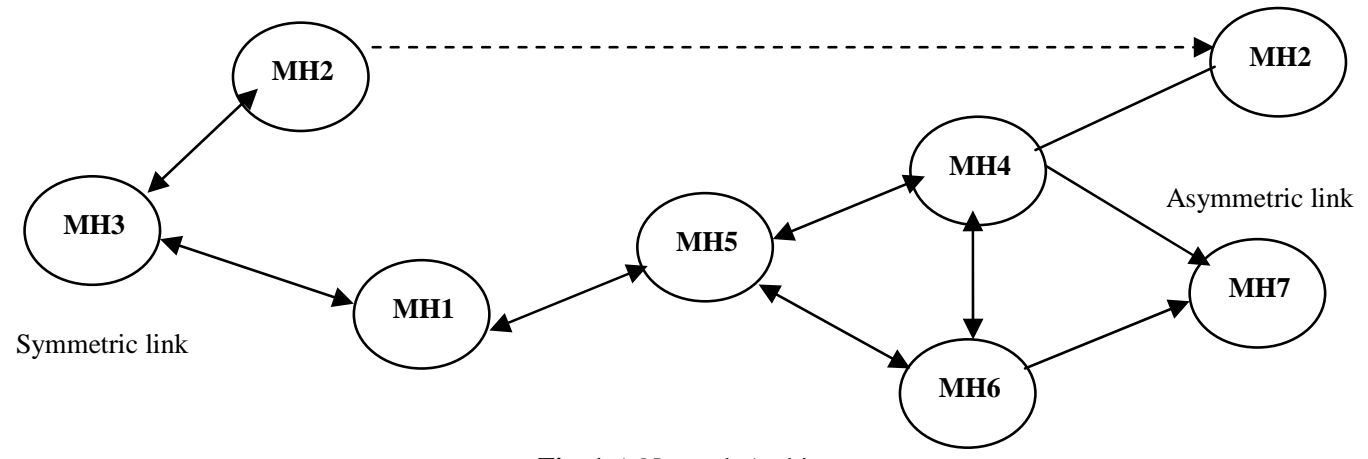

Fig. 1:A Network Architecture.

Regarding the method of operation, network systems are essentially distributed multi-bounce moveable remote systems anyplace data package are conveyed in a "store-and-forward" strategy from a source to an arbitrary end, through extraordinary nodes as appeared in Figure 1. As the MHs change, the important change in frame topology must be finished known to alternate nodes so obsolete topology data can be either refreshed or disqualified [4-5]. For instance, MH2 in Figure 1 changes its purpose of connection from $\mathrm{MH} 3$ to $\mathrm{MH} 4$, different nodes in the system should now utilize this new course to forward packets to MH2.

In Figure 1, it is accepted that it is unrealistic to have all MHs inside the scope of each other. On the off chance that all MHs are close-by inside radio range. In genuine conditions, the power expected to acquire finish network might be, in any event, infeasible, also issues, for example, battery life and spatial reusability [6]. Figure 1 raises extra issue of symmetric (bi-directional) and uneven (unidirectional) joins. As it will be seen later on, a portion of 
the conventions that consider symmetric connections with acquainted radio range, i.e., if (in Figure 1.1) MH1 is inside radio scope of $\mathrm{MH} 3$, at that point $\mathrm{MH} 3$ is additionally inside remote scope of MH1 [7].

The system is one that chances up as required, not really with any help from the current foundation or whatever other sort of settled stations [8]. This announcement can be formalized by characterizing a specially appointed system as a self-governing arrangement of portable hosts (MHs) associated by remote connections, the union of which shapes a correspondence organize demonstrated as a subjective correspondence. This is in distinction to the outstanding specific jump organize traditional that backings the requirements of remote correspondence by introducing base stations (BSs) as get to focuses.

\section{Related work}

The Limit cryptography in portable specially appointed systems consuming stochastic process the assault procedure and measurements an attack display in network. A dynamic assessment model is the consequent which can give the correct estimation of limit and keep educated time of sharing unknown the security components which function very well in usual settled system are not all that compelling without the help of any established foundation [9]. The conventional security system ordinarily require an association focus as the trust outsider to convey key administration, confirmation, get to control, multicast packets to be carried key controlling and other security offices, while it's unfeasible for portable specially appointed systems [10]. The design is acknowledged essentially in a circled mode in versatile impromptu systems various assault models in portable specially appointed systems, the majority of which concentrate on the security course and the possible assaults against a no frills arrange that plans not have any security protection [11-12].

Most assault from outer of the framework can be battled through confirmation conventions dynamic security estimation model to the scattered limit cryptography courses of action in versatile impromptu systems [13-14]. The stochastic procedure approach,Attack Stream and Attack Strength are familiar to model the attacks that the system arrangement received [16].

The mobility organization protocols can be positioned at different layers network, transport, and request the IP mobility protocol achieves mobility of a Mobile Node at the grid layer and provides network level slide, thus, the upper layers do not have to be troubled about the mobility or the significances of the IP address change IP address allocated to multiple applications exist in on a single host [18-19].

The IP address of a host system remains unpredictable in a given network as both time the system attaches to the network, a different IP address is providing by the server [20-22]. The technique for estimating the applied safety thresholds can decide more exactly whether or not eavesdropping exists in the procedure of important cipher key delivery the safety of the protocol is founded on the quantum characteristics.

The Neural networks are troubled with identifying the best sites in host image in order to implant the secret data thus successful the image quality [23-24]. The Cryptography deals with making, analyzing protocols that stop third parties from understanding secret data. The cryptography with steganography, it consequences in a controlling process which enables the social beings to communicate sensitive data over the internet securely [25]. Neural networks are used to identify the best positions with high energy constants where secret data implanting is done.

\section{Proposed system}

In now a days cryptographic algorithm need to be shared the IP address in network, It is essential for the IP address to found and uphold cryptographic entering relations. Common secret is a assessment calculated using a approved algorithm and grouping of keys. In the proposed system relating together IP configuration with enhanced IP address and the Threshold Cryptography. In usage of IP addresses reduces the problem of the limited address space. Also Threshold unicast address can be configured in network interface card through Neighbor Discovery Protocol (NDP). In this NDP approach is gives security to entire system state full neighbor framework based dynamic address allotment conventions. Our resolution trusts on a circulated then a threshold based supportive address allocation model. The newly proposed security mechanisms prevent all possible conceivable occurrences on autoconfiguration in the network.

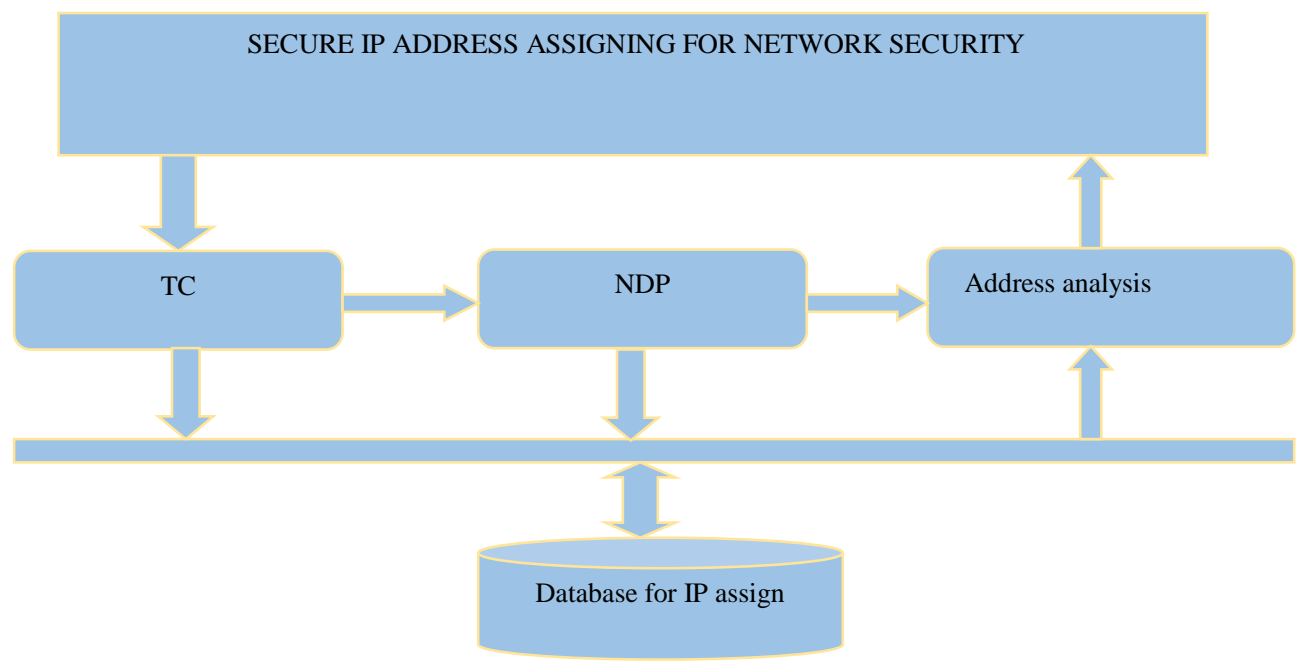

Fig. 2:Proposed System Architecture.

Figure 2, demonstrates the useful parts of the proposed approach and we will clarify each of the useful segments in detail in this area. As a rule let a1, a2, a3, a4 ... .an be the vertices and b1, b2 bm have a place with their edge set. The chart will resemble a seed of keys that will be shared by the honest to goodness clients. The Figure 3 clarifies the diagram in detail with $\mathrm{n}=5$ and $\mathrm{m}=10$. Expecting nodes have roles r1( ), r2( ), r3( ), r4( ) and r5( ) indi- vidually joined to the vertices a1, a2. a3 .a4 .a5 with b1, b2 .... $\mathrm{B} 10$ being the measured edge set of the example diagram. As the edge sets are navigated by the simulated ants the regard is passed from one node capacity to other node work. The IP address approval changes as the nodes are crossed, for instance, if underground insect directs a1 to a5 the esteem will be $\mathrm{r} 5(\mathrm{r}(\mathrm{r} 1)$ and so on. 
All the IP address period calculations manage the issues like factorization and discrete arithmetic of higher estimations of the prime numbers. These strategies are very time and helpless against the attacks. Therefore, the mathematical capacities utilized above are other than discrete and factorization sort. This is being to keep away from longer computational time and possible intrusion as is being done in before strategies talked about above. The limit examination so composed will take care of the issue as it can utilize any capacity that is touchy to the info esteems like cryptographic volumes. These capacities are invented when that node is crossed while creating the key approval.

NDP based IP address allocation

Secure IP formation anywhere IP addresses are assigned to the network nodes animatedly to useID-baseduseful IP technique. An NDP discourse allocation scheme for network either relies on the broadcasting of messages to discover the server or uses the identical address detection method. The neighbor discovery protocol is demanding an IP address for a consecutive number of times after which it allocates itself the first IP address since the known IP address block.

This address is used by a configured each and every node in the network to discover its one-hop neighbors. The NDP is used for the management of the genuine users. The procedure used is described above and the sender receives the network traffic at that occurrence. Then the timer will be used for the informing the pheromone levels. Every one minute the IP address level is efficient with the growth factor.

Every one of the means required in the key administration life cycle request the need of the irregular number generators yet the issue lies in the accessibility of such massive quantities of arbitrary numbers that don't create any impact. In the event that there are impacts a similar key will be produced and the framework will be at the hazard consequently an examination was completed for the created keys and these were tried for their pseudo irregularity.

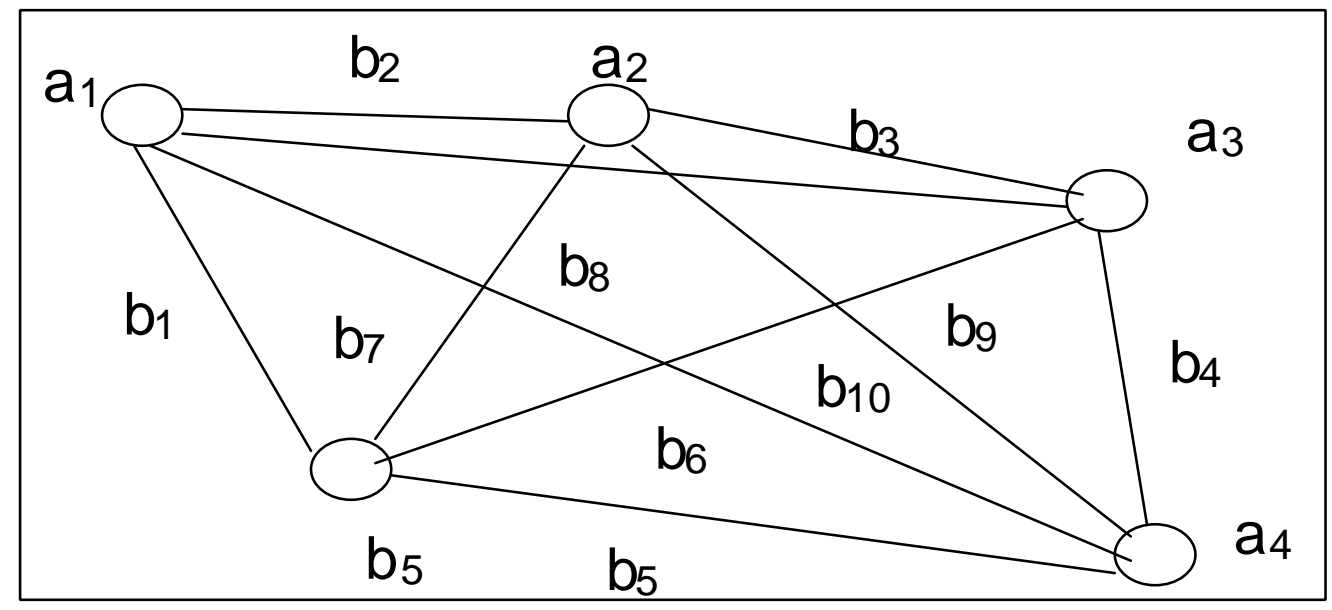

Fig. 3: 2 Example of the Cryptography Analysis.

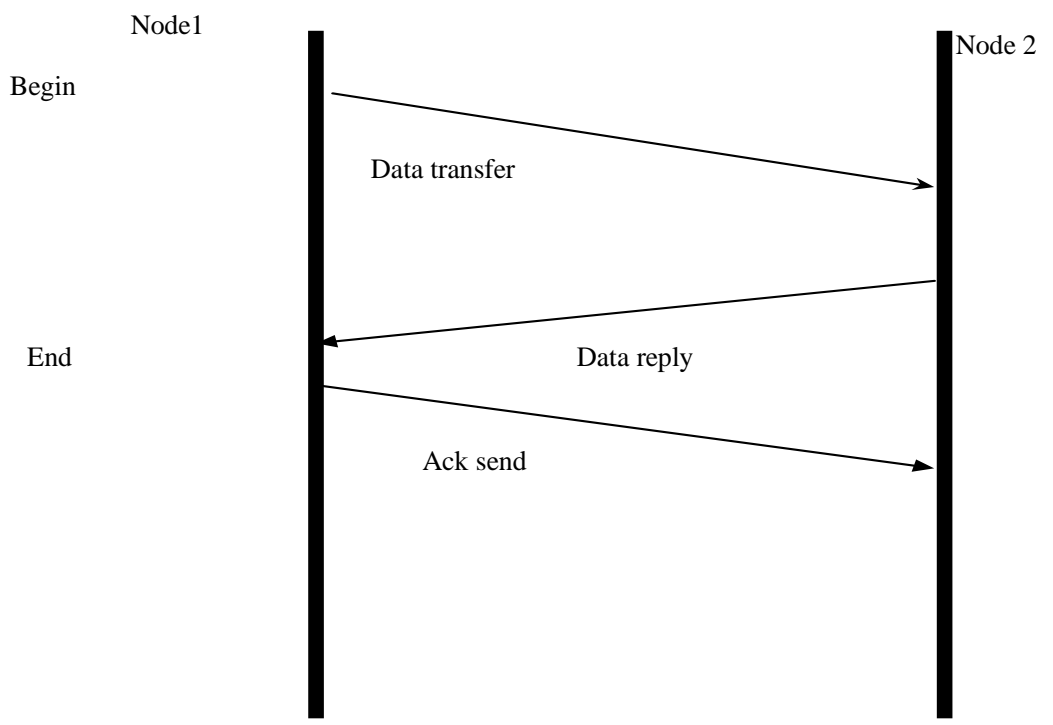

Fig. 4: 3 Time Synchronization.

\section{Algorithm}

Step 1: In address allocation procedure define an $\mathrm{msg}^{*}$ data $=$ (msg*) pkt.dataudp; for simulation process.

Step 2: Next define sink = new_node_rep; which states new node entering network as a sink node

Step 3: Next allocator= node_->ip; which states node which provides IP address

Step 4: Next public key and private key are initialized

Step 5: Next Sign which determines the offer IP address using secret key
Step 6: Next select allocator<-min_dis_tmsg*data;

Which states allocator will be chosen by a minimum distance corresponding to the new node to provide IP address.

Step 7: Next init_sink->address alloc ip0.0.1.xxx;

Which initialize the first part of IP address to a new node while entering the network.

Step 8: Next threshold value is set as 4 to all nodes which have avalidIP address and node count to 0 .

Step 9: Next config TC (up) set \$ns address alloc;

Step 10: if (threshold<sink_rep) 
config_NDP-TC+AODV;

Allocator->ip0.0.1.110.1->sink; \}

If condition checks for it's thresh-hold value, if its lesser than fourallocatorsallocateIP address else it checks for next node. The technique allocates the elliptic cryptography limitations to the recorded user, with the collection key and user-specific private key. The isolated key will be useful to decrypt the elliptic curve limitations and at each assembly the user will be sent with the new set of values which are encrypted and decrypted using the private key being distributed earlier. The proposed methods improve the efficiency of the wireless broadcast networks and increase the scalability of security in most efficient manner.

Security enhancement using TC

Ordinarily inside the network, secure communication can occur just when there are no harmful nodes. In the event that any harmful node is happening inside the network implies, its throughput gets influenced, and high security can't be accomplished. As TC approach is not doable for accomplishing full security; Threshold Cryptography approach is joined with this design to decide if the source node is a risk or not in light of the changing conditions in the system. When the number of the user increases the problem of maintaining secure access becomes complicated, and the service provider has the responsibility to maintain such access restrictions. For example, there is a situation where the medium requires the service stream to be accessed only by some set of people who belong to the organization or some specific people, where the stream is in the open spectrum. Anybody can listen to the stream which is broadcasted on the network, but they should be denied in decrypting the service.

Procedure:

Input: Network Trace Nt, Location L

Output: Null

Start

Receive Packet P.

Identify Source of packet $P$.

Saddr $=$ Source-Address $(\mathrm{P})$

Retrieve the Location from network trace.

$\mathrm{NLoc}=\mathrm{Nt}(\mathrm{Ni}(\operatorname{Loc}))$.

Verify the location with LAR.

If true then

Retrieve the previous node.

$\mathrm{L}=\mathrm{Ls}(\mathrm{Ni}) @ \mathrm{~T}_{\alpha-1}$

Compute present spaces packet details.

$\mathrm{Tpr}=\sum$ packets(Ni)€Nt(current Time Location

Compute average security as

Compute Trust node $\mathrm{Tn}=$ Tpr $\times$ Apl.

If as $>\mathrm{TTn}$ then //TTn-Trust threshold Forward packet.

End

Else

Drop the packet or look for another neighbor.

Stop.

End.

The controller, initialize parameters of elliptic and values to its different points and distributes them to the registered user. The nodes located inside the coverage of the base station or the nodes which are registered to the network or service will receive the key. The received curve features will be used to perform encryption and decryption in the later stage. Whenever the client node generates the group joins request to the controller, it sends the current sessions elliptic curve parameters and the key is selected and the stream size being used. Based on this information, the client node can perform decryption of the time.

\section{Results and discussion}

For any addressing schemes for network even in the network scenario, security enhancement, and time complexity as well as throughput of the node is increased. We therefore conduct performance analysis by comparing our proposed scheme with other schemes in the following three aspects: security, time and throughput.

Table 1:Parameter Table for Network

\begin{tabular}{ll}
\multicolumn{2}{c}{ Table 1:Parameter Table for Network } \\
\hline Number of nodes & 20 \\
\hline Channel type & Wireless Channel \\
Topology Dimension & $700 \mathrm{~m} \square 700 \mathrm{~m}$ \\
Radio range & $250 \mathrm{~m}$ \\
Node pause time & $0-200 \mathrm{~s}$ \\
MAC protocol & 802.11 \\
Maximum node speed & $10 \mathrm{~m} / \mathrm{s}, 20 \mathrm{~m} / \mathrm{s}$ and $30 \mathrm{~m} / \mathrm{s}$ \\
Traffic Pattern & CBR \\
Radio propagation model & Two ray model \\
Data payload size & 4 packets $/ \mathrm{s}$ \\
Total data rate & $327 \mathrm{kbps}$ \\
Wireless link capacity & $2000 \mathrm{kbps}$ \\
\hline
\end{tabular}

In table 1 shows the parameter for the network construction in IP addressing time then the threshold cryptography based secure IP address assigning makes use of the underlying network topology with the consideration of networks.

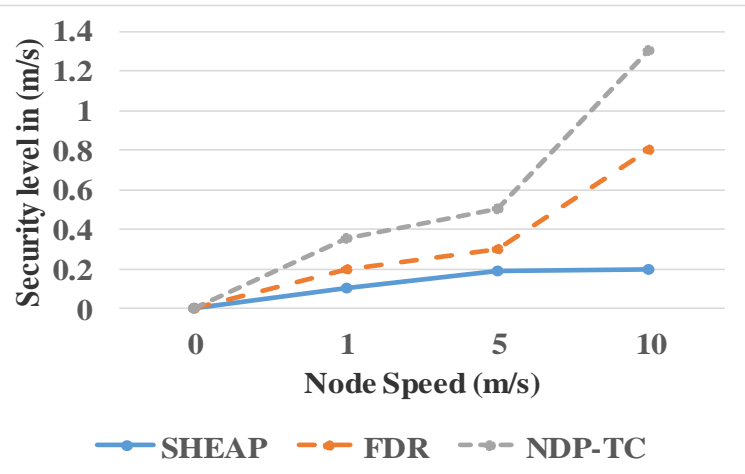

Graph 1:Comparison of Security Enhancement.

The Graph 1, shows the comparison of security enhancement produced by different methods and it shows clearly that the proposed method has provided more accuracy than others. Because Full Duplex Relay (FDR) give $89.9 \%$ but our proposed system gives $93.35 \%$ for security in data transmission.

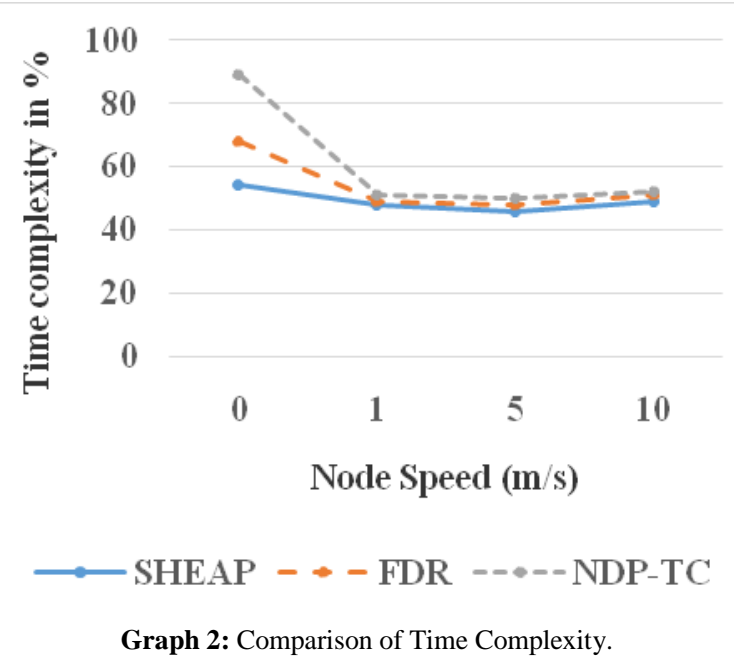

The Graph 2, shows the time complexity produced by different methods in performing security of the network and it shows clearly that the proposed method has produced less time complexity 
than other methods, also our proposed method shows the $19.3 \mathrm{~ms}$ less time complexity compare to FDR methods.

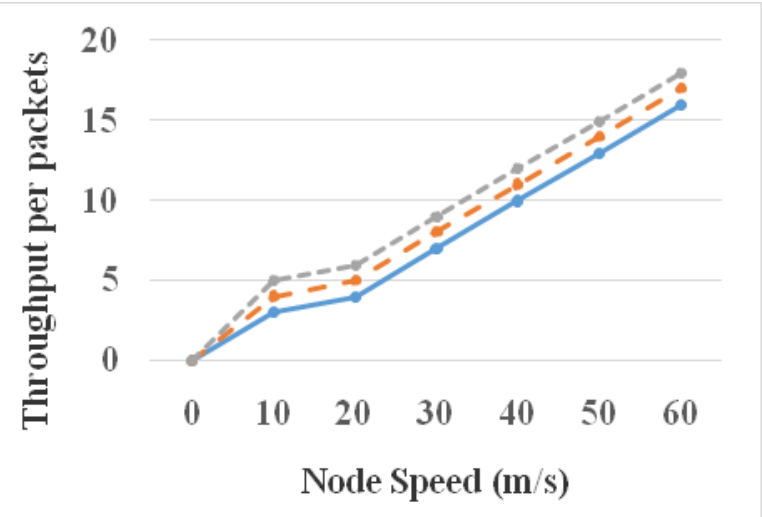

$\longrightarrow$ SHEAP $-\rightarrow$ FDR $-\cdots--$ NDP-TC

Graph 3: Comparison of Throughput Performance.

The Graph 3, shows the similar result of return ratio formed by different methods and it demonstrations clearly that the planned approach has produced more performance than other methods. The network will generate NDP materials via TC generation and file preprocess, and then upload the data to the datacenter. Different from previous schemes, the user will store a remote method instead of a network server as metadata. Moreover, the client will authorize the NDP a value signature authentication verify Data updating: the TC performs the client's fine-grained update requests via access role of data, then the user request.

\section{Conclusion}

This paper tryes to proposes the new method threshold cryptography which provide security for network based on IPaddress distribution. The new proposed security systems keep every single conceivable assault on auto-design specifically organize. The cost of the security change brought by TC is a bit of expanding in inertness and correspondence overhead, which stays satisfactory. While NDP is sorted out, the developing in correspondence overhead and dormancy is very sensible. Finally in our proposed system achieved the high security depends on all another factor so automatically increase the overall network performance up to $98.56 \%$ as well as delivery ratio will increase in to $96.78 \%$ in network.

\section{References}

[1] Dong Q, Liu D \& Wright M, "Mitigating jamming attacks in wireless broadcast systems", Wireless networks, Vol.19, No.8, (2013), pp.1867-1880.https://doi.org/10.1007/s11276-013-0574-0.

[2] Rajesh KV, "An Efficient Key Management Scheme for Secure Data Access Control in Wireless Broadcast Services", international Journal of Smart Sensors and Ad Hoc Networks, Vol.1, No.4, (2012).

[3] Yu H, He J, Zhang T, Xiao P \& Zhang Y, "Enabling end-to-end secure communication between wireless sensor networks and the Internet", World Wide Web, Vol.16, No.4, (2013), pp.515540.https://doi.org/10.1007/s11280-012-0194-0.

[4] Cao X, Kou W, Zeng X \& Dang L, "Identity-based anonymous remote authentication for value-added services in mobile networks", IEEE Trans. Veh. Technol., Vol.58, No.7, (2009), pp.35083517.https://doi.org/10.1109/TVT.2009.2012389.

[5] Christophe B, Boussard M, Lu M, Pastor A \&Toubiana V, "The web of things vision: Things as a service and interaction patterns", Bell labs technical journal, Vol.16, No.1, (2011), pp.5561.https://doi.org/10.1002/bltj.20485.

[6] Granjal J, Monteiro E \& Silva JS, "A Secure interconnection model for IPv6 enabled wireless sensor networks", Proceeding of the IFIP
Wireless

Days,

6.https://doi.org/10.1109/WD.2010.5657743.

pp.1-

[7] Gupta V, Poursohi A \& Udupi P, "Sensor network: an open data exchange for the web of things", Proceeding of the 8th IEEE International Conference on Pervasive Computing and Communications Workshops, (2010), pp.753-755.

[8] Mzid R, Boujelben M, Youssef H \& Abid M, “Adapting TLS handshake protocol for heterogeneous IP-based WSN using identity based cryptography", Proceeding of the International Conference on Wireless and Ubiquitous Systems, (2010), pp.18.https://doi.org/10.1109/ICWUS.2010.5671367.

[9] Roman R, Alcaraz C, Lopez J \&Sklavos N, "Key management systems for sensor networks in the context of the Internet of Things", Computers \& Electrical Engineering, Vol.37, No.2, (2011), pp.147159.https://doi.org/10.1016/j.compeleceng.2011.01.009.

[10] $\mathrm{Yu} \mathrm{H} \mathrm{\&} \mathrm{He} \mathrm{J,} \mathrm{"Trust-based} \mathrm{mutual} \mathrm{authentication} \mathrm{for} \mathrm{bootstrapping}$ in 6LoWPAN", J. Commun. Technol., Vol.7, No.8, (2012), pp.634642.https://doi.org/10.4304/jcm.7.8.634-642.

[11] Ferng HW, Nurhakim J \&Horng SJ, "Key management protocol with end-to-end data security and key revocation for a multi-BS wireless sensor network", Wireless networks, Vol.20, No.4, (2014), pp.625-637.https://doi.org/10.1007/s11276-013-0627-4.

[12] Zhao S, "A key management and secure routing integrated framework for Mobile Ad-hoc Networks", Elsevier, Ad Hoc Networks, Vol.11, No.3, (2013), pp.10461061.https://doi.org/10.1016/j.adhoc.2012.11.005

[13] ChuahM \& Yang P, "Performance evaluation of content-based information retrieval schemes for DTNs", Proc. IEEE MILCOM, (2007), pp.1-7.https://doi.org/10.1109/MILCOM.2007.4455020.

[14] Kallahalla M, Riedel E, Swaminathan R, Wang Q \& Fu K, "Plutus: Scalable secure file Hasingring on untrusted storage", Proc. Conf. File Storage Technol., (2003), pp.29-42.

[15] Ibraimi L, Petkovic M, Nikova S, Hartel P \& Jonker W, "Mediated ciphertext-policy attribute-based encryption and its application", International Workshop on Information Security Applications, (2009),pp.309-323.https://doi.org/10.1007/978-3-642-10838-9_23.

[16] Liu Y, Ning P, Dai H \& Liu A, "Randomized differential DSSS: Jamming-resistant wireless broadcast communication", Proceedings of the 29th IEEE international conference on computer communications,

(2010).https://doi.org/10.1109/INFCOM.2010.5462156.

[17] Liu D, Raymer J \& Fox A, "Efficient and timely jamming detection in wireless sensor networks", Proceedings of IEEE international conference on mobile ad hoc and sensor systems (MASS), (2012).https://doi.org/10.1109/MASS.2012.6502533.

[18] Zhang Y, Wu C, Cao J \& Li X, "A secret sharing-based key management in hierarchical wireless sensor network", International Journal of Distributed Sensor Networks, Vol.9, No.6, (2013).https://doi.org/10.1155/2013/406061.

[19] Bertier M, Mostefaoui A \&Trédan G, "Low-cost secret-sharing in sensor networks,"Proceedings of the IEEE 12th International Symposium on High Assurance Systems Engineering, (2010), pp. 1-9. https://doi.org/10.1109/HASE.2010.16.

[20] Claveirole T, Dias De Amorim M, Abdalla M \& Viniotis Y, "Securing wireless sensor networks against aggregator compromises", IEEE Communications Magazine, Vol.46, No.4, (2008), pp.134-141.https://doi.org/10.1109/MCOM.2008.4481352.

[21] Seyed HN, Amir HJ \& Vanesa D, "A distributed group rekeying scheme for wireless sensor networks", Proc.of the 6th International Conference on Systems and Networks Communications, (2011), pp.127-135.

[22] Zhang YY, Li XZ, Liu JM, Yang JC \& Cui BJ, “A secure hierarchical key management scheme in wireless sensor network," The International Journal of Distributed Sensor Networks, (2012) https://doi.org/10.1155/2012/547471.

[23] Zhang YY, Li XZ, Yang JC, Liu YA, Xiong NX \&Vasilakos AV, "A real-time dynamic key management for a hierarchical wireless multimedia sensor network", Multimedia Tools and Applications, (2012).

[24] Agrawal S, "Verifiable secret sharing in a total of three rounds", Information Processing Letters, Vol.112, (2012), pp.856859.https://doi.org/10.1016/j.ipl.2012.08.003.

[25] Hua C, Liao X \& Cheng X, "Verifiable multi-secret sharing based on LFSR sequences", Theoretical Computer Science, Vol.445, (2012), pp.52-62.https://doi.org/10.1016/j.tcs.2012.05.006. 